\title{
A study of $\tilde{Y}$-transform
}

\author{
Padama Kumawat ${ }^{1 *}$ and Yogesh Khandelwal ${ }^{2}$
}

\begin{abstract}
In this paper we introduce and study an integral transform ( $\tilde{Y}$-transform) whose kernel is the $D_{\mu, \rho}^{v}(z)$ function which is generalized form of Kratzel function introduced by Kratzel [10]. First, we obtain the basic properties of $\tilde{Y}$ transform. Further, we establish connection formulae of $\tilde{Y}$-transform with Mellin transform, Laplace transform and Saigo operators. Next, we find the images of the product of $H$-function and $S_{V}^{U}$ under this transform.

\section{Keywords}

Mellin transform, Laplace transform, Saigo operators.

\section{AMS Subject Classification}

$46 \mathrm{~F} 12$.

1,2 Department of Mathematics, Maharshi Arvind University, Jaipur-302017, India.

*Corresponding author: ${ }^{1}$ yogeshmaths81@gmail.com

Article History: Received 24 March 2019; Accepted 19 June 2019

(C)2019 MJM.
\end{abstract}

\section{Contents}

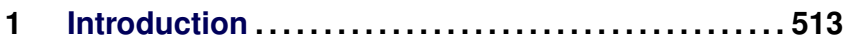

2 Mellin transform and Laplace transform .........514

3 Right-hand sided Riemann-Liouville fractional operators and $\tilde{Y}$-transform ......................... 515

4 Saigo transform and $\tilde{Y}$-transform .............516

5 Conclusion ................................ 517

References ............................. 517

\section{Introduction}

We introduce and study the following integral transform $(\tilde{Y}$-transform)

$$
\tilde{Y}_{v}^{\mu, \rho}[g(z): x]=\int_{0}^{\infty} D_{\mu, \rho}^{v}(z x) g(z) d z, \quad x>0
$$

$D_{\mu, \rho}^{v}$ function occurring in (1.1) is generalized form of Kratzel function introduced by Dernek [16] defined as

$$
D_{\mu, \rho}^{v}(z)=\int_{0}^{\infty} y^{v-1} e^{-y^{\rho}-z y^{-\mu}} d y
$$

where $z>0, \mu>0, \rho \in \mathbb{R}, v \in \mathbb{C}$.

For $\mu=1,(1.1)$ reduces to Kratzel transform [[9], p.604, Eq.(5)] defined in the following manner

$$
\tilde{Y}_{v}^{\rho}[g(z): x]=K_{v}^{\rho}[g(z): x]=\int_{0}^{\infty} D_{\rho}^{v}(z) g(z) d z, \quad x>0
$$

where $D_{\rho}^{v}(z)$ is Kratzel function studied by Kratzel [10,p.603 , Eq.(2)] as

$$
D_{\rho}^{v}(z)=\int_{0}^{\infty} y^{v-1} e^{-y^{\rho}-z y^{-1}} d y,
$$

where $z, \rho$ and $v$ as same mentioned in (1.2).

If $\mu=1, \rho=1$, our transform of (1.1) reduces to Meijer's transform. When $\rho=1$ and $z=\frac{t^{2}}{4}$ then the Kratzel function $D_{\rho}^{\nu}(z)$ is related to the Bessel modified function of the second kind $K_{v}(z)$ defined as follows:

$$
D_{1}^{v}\left(\frac{t^{2}}{4}\right)=2\left(\frac{t}{2}\right) K_{-v}(t)
$$

The $\tilde{T}$-transform investigated by R. Jain et al. [17] expressed as follows

$$
\tilde{T}_{v, w, b}^{\rho, \mu, \alpha}[g(z) ; x]=\int_{0}^{\infty} I_{3}(z x, w, v, \rho, \mu, b, \alpha) g(z) d z .
$$

Here $I_{3}(z, w, v, \rho, \mu, b, \alpha)$ function is generalization of astrophysical thermonuclear function introduced by Saxena [[18], p.35, Eq.(4.1)] defined as

$$
\begin{aligned}
& I_{3}(z, w, v, \rho, \mu, b, \alpha) \\
& =\int_{0}^{\infty} y^{v-1}\left[1+b(\alpha-1) y^{\rho}\right]^{-\frac{1}{(\alpha-1)}} e^{-z(y+w)^{-\mu}} d y .
\end{aligned}
$$

When $t=0$ above function lead to generalized form of Kratzel function $D_{\rho, \mu}^{v, \alpha}(z)$

$$
\lim _{t \rightarrow 0} I_{3}(z, w, \nu, \rho, \mu, b, \alpha)=D_{\rho, \mu}^{\nu, \alpha}(z) .
$$


If $w=0$ in (1.7), we have

$$
\lim _{\alpha \rightarrow 1} D_{\rho, \mu}^{v, \alpha}(z)=D_{\mu, \rho}^{v}(z),
$$

where $D_{\mu, \rho}^{v}(z)$ is generalized Kratzel function studied by Dernek [[16], Eq.(5)] which is the kernel of our transform of study. The function is generalization of Kratzel function since

$$
\lim _{\mu \rightarrow 0} D_{\mu, \rho}^{v}=D_{\rho}^{v}(z)
$$

If $w=0$, the transform defined by (1.6) reduces to $P$-transform studied by Dilip Kumar [[8], p.603, Eq.(1)], which is given by

$$
P_{v, \alpha}^{\rho, \mu}[g(z) ; x]=\int_{0}^{\infty} D_{\mu, \rho}^{v, \alpha}(z x) g(z) d z, \quad x>0
$$

where $D_{\mu, \rho}^{v, \alpha}(z)$ is generalized Kratzel function studied by Dilip Kumar [[9], p.603, Eq.(2)]. When (1.6) reduces to transform of our study

$$
\tilde{Y}_{v}^{\rho, \mu}[g(z): x]=\int_{0}^{\infty} D_{\mu, \rho}^{v}(z x) g(z) d z, \quad x>0
$$

The transform introduced by Kratzel [9] and its several generalization were investigated by many authors. Bonilla et al. [7] studied the Kratzel transform in the space $F P, \mu$ and $F P, \mu 1$ Glaeske et al. [10] introduced a modified version of the Kratzel transform and its compositions with fractional calculus operators on the spaces of $F P, \mu$ and $F P, \mu 1$. Kilbas et al. [5] obtained the asymptotic representation for the modified Kratzel function, Liouville and Erdelyi-Kober type fractional integrals of the modified Kratzel function. Kilbas et al. [6] studied the Kratzel function in (1.4) for all values of $\frac{1}{2}$ and established it in terms of Fox's $H$-function.

This paper is organized as follows: First, we introduce a new class of integral transform, whose Kernel is the generalization of Kratzel function. First, we present the basic properties of $\tilde{Y}$ - transform and then establish composition formulae of $\tilde{Y}$-transform with Mellin transform, Laplace transform and Saigo operators. Next, we find the images of the product of $H$-function and $S_{V}^{U}$ under this transform.

\section{Some properties of $\tilde{Y}_{v}^{\rho, \mu}$-transform}

In this section, we will give some properties of transform of our study.

\section{Linear Property:}

Let $g_{1}(z), g_{2}(z)$ be two functions and $c_{1}, c_{2}$ be arbitrary constants such that

$$
\begin{aligned}
& \tilde{Y}_{v}^{\rho, \mu}\left[\left(c_{1} g_{1}\right)(z) ; x\right]=c_{1} \int_{0}^{\infty} D_{\rho, \mu}^{v}(z x) g_{1}(z) d z \\
& \tilde{Y}_{v}^{\rho, \mu}\left[\left(c_{2} g_{2}\right)(z) ; x\right]=c_{2} \int_{0}^{\infty} D_{\rho, \mu}^{v}(z x) g_{2}(z) d z,
\end{aligned}
$$

then

$$
\begin{aligned}
\tilde{Y}_{v}^{\rho, \mu}\left[\left(c_{1} g_{1}+c_{2} g_{2}\right) ; x\right]= & c_{1} \tilde{Y}_{v}^{\rho, \mu}\left[g_{1}(z) ; x\right] \\
& +c_{2} \tilde{Y}_{v}^{\rho, \mu}\left[g_{2}(z) ; x\right] .
\end{aligned}
$$

\section{Shifting Property:}

If $g(z)$ is a function such that $\tilde{Y}_{v}^{\rho, \mu}[g(z): x]=\tilde{g}(x)$ then

$$
\tilde{Y}_{v}^{\rho, \mu}\left[e^{-b z y^{-\mu}} g(z) ; x\right]=\tilde{g}(x+b) .
$$

To prove (1.16), expressing $\tilde{Y}$-transform with the help of (1.1) and (1.2), we get the following from say $(\Delta)$

$$
\Delta=\int_{0}^{\infty}\left\{\int_{0}^{\infty} y^{v-1} e^{-y^{\rho}} e^{-(x+b) z(y+w)^{-\mu}} d y\right\} g(z) d z .
$$

Using (1.2), we write the inner integral in terms of the kernel, we get the required result.

$m^{\text {th }}$ derivative of $\tilde{Y}_{\rho, \mu}^{v}$-transform

$$
D^{m}\left(\tilde{Y}_{v}^{\rho, \mu}(g(z): x)\right)=\tilde{Y}_{v-\mu m-r}^{\rho, \mu}\left((-z)^{m} g(z) ; x\right) \text {. }
$$

In order to prove (1.18), we write $\tilde{Y}$-transform with the help of (1.1) and (1.2) in the left hand side of it. Next, we change the order of differentiation and integration which is permissible here under the condition stated. We get the following form say $(\Delta)$

$$
\Delta=\int_{0}^{\infty} \int_{0}^{\infty} y^{\nu-1} e^{-y^{\rho}} \frac{d^{m}}{d x^{m}}\left(e^{-z x y^{-\mu}}\right) g(z) d y d z
$$

after differentiating $m$ times to above equation, we write the result obtained in terms of $\tilde{Y}_{v}^{\rho, \mu}$ after a little simplification. We get the desired result.

\section{Mellin transform and Laplace transform}

The Mellin transform of the function is defined as follows

$$
M\{f(z) ; s\}=\int_{0}^{\infty} z^{s-1} f(z) d z, \quad s \in \mathbb{C}, z>0
$$

Theorem 2.1. Let $g(z) \in L_{v, r}(0, \infty), \rho \in \mathbb{R}, \rho \neq 0, s, v \in \mathbb{C}, \mu>$ $0, z>0$ such that $\operatorname{Re}(s)>0, \operatorname{Re}\left(\frac{v+\mu s}{\rho}\right)>0$ and $M\left\{\tilde{Y}_{v}^{\rho, \mu}\{g(z)\} ; s\right\}$ and $M\{g(z) ; s\}$ exist then we have the following result

$$
M\left[\tilde{Y}_{v}^{\rho, \mu}\{g(z)\} ; s\right]=\frac{\Gamma s}{|\rho|} \Gamma\left(\frac{v+\mu s}{\rho}\right) M\{g(z) ; 1-s\},
$$

where $M\{g(z) ; s\}$ is the well known Mellin-transform of function $g(z)$.

Proof. Using (1.1) and (2.1), we have

$$
\begin{aligned}
& M\left[\tilde{Y}_{v}^{\rho, \mu}\{g(z)\} ; s\right] \\
& =\int_{0}^{\infty} g(z)\left\{\int_{0}^{\infty} x^{\nu-1}\left\{\int_{0}^{\infty} y^{\nu-1} e^{-y^{\rho}-z x y^{-\mu}} d y\right\} d x\right\} d z .
\end{aligned}
$$


Changing the order of integration and using the substitution of $x y^{-\mu}=u$, we have

$$
\begin{aligned}
& M\left[\tilde{Y}_{v}^{\rho, \mu}\{g(z)\} ; s\right] \\
& =\int_{0}^{\infty} g(z)\left\{\int_{0}^{\infty} y^{\nu-1} e^{-y^{\rho}}\left\{\int_{0}^{\infty} x^{s-1} e^{-z x y^{-\mu}} d y\right\} d x\right\} d z .
\end{aligned}
$$

Applying the known formula from [[1], p.145], we find that

$$
M\left[\tilde{Y}_{v}^{\rho, \mu}\{g(z)\} ; s\right]=\frac{\Gamma s}{\rho} \int_{0}^{\infty} g(z)\left\{\int_{0}^{\infty} t^{\frac{v+\mu s}{\rho}-1} e^{-t} d t\right\} d z .
$$

When $\rho>0$, we have

$$
M\left[\tilde{Y}_{v}^{\rho, \mu}\{g(z)\} ; s\right]=\frac{\Gamma s}{|\rho|} \Gamma\left(\frac{v+\mu s}{\rho}\right) M\{g(z) ; 1-s\} .
$$

Corollary 2.2. If we take $\mu=1$ and the conditions of Theorem 2.1 are satisfied, then

$$
\begin{aligned}
& M\left[\tilde{Y}_{v}^{\rho, 1}\{g(z)\} ; s\right]=M\left[K_{v}^{\rho}\{g(z) ; x\} ; s\right] \\
& =\frac{\Gamma s}{|\rho|} \Gamma\left(\frac{v+s}{\rho}\right) M\{g(z) ; 1-s\} .
\end{aligned}
$$

Laplace Transform: The Laplace transform of a function $g$ is defined as follows:

$$
L\{g(z) ; z\}=\int_{0}^{\infty} e^{-s z} g(z) d z, \quad s \in \mathbb{C}, z>0 .
$$

For $\rho>0$, we have

$$
\begin{aligned}
& L\left[\tilde{Y}_{\rho, \mu}^{v}\{g(z) ; x\} ; s\right] \\
& =\frac{1}{s \rho} \int_{0}^{\infty} H_{2,1}^{1,2}\left[\begin{array}{c}
(0,1) \\
s
\end{array} \mid(0,1),\left(\frac{v}{\rho}, \frac{\mu}{\rho}\right)\right] g(z) d z
\end{aligned}
$$

and for $\rho<0$, we have

$$
\begin{aligned}
& L\left[\tilde{Y}_{\rho, \mu}^{v}\{g(z) ; x\} ; s\right] \\
& =-\frac{1}{s \rho} \int_{0}^{\infty} H_{2,1}^{1,2}\left[\begin{array}{c}
\left.\frac{z}{s} \mid(0,1),\left(1-\frac{v}{\rho}, \frac{\mu}{\rho}\right)\right] g(z) d z . \\
(0,1)
\end{array}\right]
\end{aligned}
$$

Corollary 2.3. If we take $\mu=1$ and the conditions of Theorem 2.2 are satisfied, then we get the Laplace transform of Kratzel transform given in [9]. Further, if $\mu=1, \rho=1$ in Theorem 2.2, we get the Laplace transform of Meijer transform.

Proof. Using (1.1) and (2.8), we have

$$
\begin{aligned}
& L\left[\tilde{Y}_{v}^{\rho, \mu}\{g(z) ; x\} ; s\right] \\
& =\int_{0}^{\infty} e^{-s x} \int_{0}^{\infty} D_{\mu, \rho}^{v}(z x) f(z) d z d x \\
& =\int_{0}^{\infty} e^{-s x} \int_{0}^{\infty} \int_{L} \Gamma(-\zeta) \Gamma\left(\frac{v+\mu \zeta}{\rho}\right)(z x)^{\zeta} f(z) d z d x .
\end{aligned}
$$

Changing the order of integration which is possible because of the uniform continuity of the integral and using gamma function, we get

$$
\begin{aligned}
& L\left[\tilde{Y}_{v}^{\rho, \mu}\{g(z) ; x\} ; s\right]=\frac{1}{2 \phi i \rho} \int_{L} \Gamma(-\zeta) \Gamma\left(\frac{v+\mu \zeta}{\rho}\right) \\
& (\times) \int_{0}^{\infty} z^{\zeta} f(z)\left\{e^{-s x}(x)^{\zeta} d x\right\} d z \\
& =\frac{1}{2 \phi i \rho} \int_{L} \frac{1}{s^{\zeta+1}} \Gamma(-\zeta) \Gamma\left(\frac{v+\mu \zeta}{\rho}\right) \\
& (\times) \Gamma(1+\zeta)\left\{z^{\zeta} f(z) d z\right\} d \zeta,
\end{aligned}
$$

where $\operatorname{Re}(s)>0, \operatorname{Re}\left(\frac{v+\mu s}{\rho}\right)>0$.

\section{Right-hand sided Riemann-Liouville fractional operators and $\tilde{Y}$-transform}

The Liouville fractional integral is defined by

$$
\left(\mathscr{F}^{\alpha}\right)(x)=\frac{1}{\Gamma(\alpha)} \int_{s}^{\infty}(t-x)^{\alpha-1} g(t) d t
$$

and its derivative $\mathscr{F}_{-}^{\alpha}$ and $D_{-}^{\alpha}$ are

$$
\begin{aligned}
& \left(D_{-} g\right)(x)=\left(-\frac{d}{d x}\right)^{[\operatorname{Re}(\alpha)+1]}\left(\mathscr{F}^{1-\alpha+\operatorname{Re}(\alpha)}\right)(x) \\
& =\frac{1}{\Gamma(1-\alpha+\operatorname{Re}(\alpha))} \int_{x}^{\infty}(t-x)^{-\alpha+\operatorname{Re}(\alpha)} g(t) d t,
\end{aligned}
$$

where $x>0, \alpha \in \mathbb{C}, \operatorname{Re}(\alpha)>0$.

Theorem 3.1. Let $g(z) \in L_{v, r}(0, \infty), \rho \in R, \rho \neq 0, s, v \in \mathbb{C}$ and when $\rho>0$, then we have the following result

$$
\mathscr{F}_{-}^{\alpha}\left[\tilde{Y}_{\rho, \mu}^{v} g(z) ; x\right]=\left[\tilde{Y}_{\rho, \mu}^{v+\gamma \mu} z^{-\gamma} g(z) ; x\right] .
$$

Proof. Using (1.1) and (3.1), we have

$$
\begin{aligned}
& \mathscr{F}_{-}^{\alpha}\left[\tilde{Y}_{\rho, \mu}^{\nu} g(z) ; x\right] \\
& =\int_{0}^{\infty} g(z)\left\{\int_{x}^{\infty}(t-x)^{\alpha-1}\left\{y^{\nu-1} e^{-y^{\rho}-z x y^{-\mu}} d y\right\} d t\right\} d z \\
& =\int_{0}^{\infty} g(z) \int_{0}^{\infty} y^{\nu-1} e^{-y^{\rho}} \\
& (\times)\left\{\int_{s}^{\infty}(t-x)^{\alpha-1} e^{-z t y^{-\mu}} d x\right\} d y d z
\end{aligned}
$$

Applying well known formula

$$
\begin{aligned}
& \mathscr{F}_{-}^{\alpha}\left(e^{-\gamma x}\right)=\gamma^{\alpha} e^{-\gamma t}, \quad \operatorname{Re}(\alpha)>0, \operatorname{Re}(\gamma)>0 \\
& =\int_{0}^{\infty} g(z) z^{-\lambda}\left\{\int_{0}^{\infty} y^{v+\mu \alpha-1} e^{-y^{\rho}-z x y^{-\mu}} d y\right\} d z \\
& =\int_{0}^{\infty} g(z) z^{-\lambda} \tilde{Y}_{\rho, \mu}^{v+\alpha \mu}(z x) d z=\tilde{Y}_{\rho, \mu}^{v+\alpha \mu}\left\{z^{-\lambda} g(z) ; x\right\} .
\end{aligned}
$$


Theorem 3.2. Let $g(z) \in L_{v, r}(0, \infty), s, v \in \mathbb{C}, \rho \in R, \rho \neq 0$ and when $\rho>0$, then we have the following result

$$
D_{-}^{\alpha}\left[\tilde{Y}_{\rho, \mu}^{v} g(z) ; x\right]=\left[\tilde{Y}_{\rho, \mu}^{\nu-\alpha \mu} z^{-\gamma} g(z) ; x\right] .
$$

Proof. Using (1.1) and (3.2), we have

$$
\begin{aligned}
& D_{-}^{\alpha}\left[\tilde{Y}_{v}^{\rho, \mu}\{g(z) ; x\} ; s\right] \\
& =\left(-\frac{d}{d x}\right)^{m} I^{m-\alpha}\left[\tilde{Y}_{\rho, \mu}^{v}\{g(z) ; x\}\right] \\
& =\left(-\frac{d}{d x}\right)^{n} \int_{0}^{\infty} g(z) z^{-(n-\alpha)}\left\{\int_{0}^{\infty} y^{v-1} e^{-y^{\rho}-z t y^{-\mu}} d y\right\} d z \\
& =\int_{0}^{\infty} g(z) z^{\alpha}\left\{y^{v-\mu \alpha-1} e^{-y^{\rho}-z t y^{-\mu}} d y\right\} d z \\
& =\int_{0}^{\infty} \tilde{Y}_{\rho, \mu}^{v-\mu \alpha, \alpha}(z x) z^{\alpha} g(z) d z .
\end{aligned}
$$

and

$$
\begin{aligned}
& I_{+}^{\gamma, \delta, \kappa}[g(t), x]=\frac{1}{\Gamma(\alpha)} \int_{x}^{\infty}(t-x)^{\gamma-1} t^{-\gamma-\delta} \\
& { }_{2} F_{1}\left(\gamma+\delta,-\kappa ; \gamma ; 1-\frac{x}{t}\right) g(t) d t, x>0
\end{aligned}
$$

where ${ }_{2} F_{1}(\alpha, \beta, \lambda ; z)$ is Gauss Hyergeometric series defined in [1] for $\lambda \neq 0,-1,-2$.

When $\delta=-\gamma$, the above equation (4.1) and (4.2) reduce to the classical Riemann-Liouville fractional integral operators [[19], p.94, Eqs.(5.1) \& (5.3)].

Further

$$
\begin{aligned}
D_{+}^{\gamma, \delta, \kappa}[g(t), x]= & \left(\frac{d}{d t}\right)^{n} I_{+}^{-\gamma+n,-\delta-n, \gamma+\kappa-n}[g(t), x], \\
& (\operatorname{Re}(\gamma) \geq 0, n=\operatorname{Re}[(\gamma)]+1)
\end{aligned}
$$

and

$$
\begin{aligned}
D_{-}^{\gamma, \delta, \kappa}[g(t), x]= & \left(-\frac{d}{d t}\right)^{n} I_{+}^{-\gamma+n,-\delta-n, \gamma+\kappa-n}[g(t), x] \\
& (\operatorname{Re}(\gamma) \geq 0, n=\operatorname{Re}(\gamma)+1)
\end{aligned}
$$

where $\operatorname{Re}(\gamma) \geq 0, \gamma, \delta, \kappa \in R$ and $I_{+}^{\gamma, \delta, \kappa}, I_{-}^{\gamma, \delta, \kappa}$ known as generalized fractional integral operators given by (4.1) and (4.2) respectively.
When $\delta=-\gamma$, the above equations (4.3) and (4.4) reduces to the classical Riemann-Liouville fractional differential operators [[4], p. 80, Eqs.(2.2.3-2.2.4)].

Again, if $\delta=0$, equations (4.3) and (4.4) Erdelyi-Kober fractional differential operators [[6], p.109, Eqs.(2.6.35-2.6.36)]. Required Results: Known results which are required to establish the connections of Saigo transform and our transform of study as follows:

Result 1: [[3], p. 871, Eqn. (15)]

$$
I_{+}^{\gamma, \delta, x}\left[t^{\alpha-1} ; x\right]=\frac{\Gamma(\kappa+\sigma-\delta) \Gamma(\sigma)}{\Gamma(\sigma-\delta) \Gamma(\sigma+\kappa+\gamma)} x^{\sigma-\delta-1},
$$

where $\operatorname{Re}(\gamma)>0, \operatorname{Re}(\sigma)>\max \{0, \operatorname{Re}(\delta-\kappa)\}, \kappa, \gamma, \delta \in R$.

Result 2: [[3], p. 872, Eqn. (21)]

$$
I_{-}^{\gamma, \delta, x}\left[t^{\alpha-1} ; x\right]=\frac{\Gamma(1+\delta-\sigma) \Gamma(1+\kappa-\sigma)}{\Gamma(1-\sigma) \Gamma(1+\gamma+\delta+\kappa-\sigma)} x^{\sigma-\delta-1},
$$

where $I_{+}^{\gamma, \delta, x}$ and $I_{-}^{\gamma, \delta, x}$ occurring in (4.5) and (4.6) are known as Saigo fractional integral operators given by (4.1) and (4.2).

Result 3: [[2], p. 327, Eqn. (22)]

$$
D_{+}^{\gamma, \delta, \kappa}\left[t^{\alpha-1} ; x\right]=\frac{\Gamma(\sigma) \Gamma(\delta+\sigma+\gamma+\kappa)}{\Gamma(\sigma+\kappa) \Gamma(\sigma+\delta)} x^{\sigma+\delta-1}, x>0
$$

Result 4: [[2], p. 328, Ean. (26)]

$$
\begin{aligned}
& D_{-}^{\gamma, \delta, \kappa}\left[t^{\alpha-1} ; x\right] \\
& =\frac{\Gamma(1-\sigma-\delta) \Gamma(1-\sigma+\gamma+\kappa)}{\Gamma(1-\sigma) \Gamma(1-\sigma+\kappa-\delta)} x^{\sigma+\delta-1}, x>0
\end{aligned}
$$

where $\operatorname{Re}(\gamma) \geq 0, \operatorname{Re}(\sigma)>-\min \{0, \operatorname{Re}(\gamma+\delta+\kappa)\}$.

Here $D_{+}^{\gamma, \delta, \kappa}$ and $D_{-}^{\gamma, \delta, \kappa}$ occurring in (4.7) and (4.8) are known as Saigo fractional integral operators given by (4.3) and (4.4).

Theorem 4.1. Let $g(z) \in L_{v, r}(0, \infty), s, v \in \mathbb{C}, \rho \in R, \rho \neq 0$, then we have the following results:

$$
\begin{aligned}
& I_{0, x}^{\gamma, \delta, \kappa}\left[\tilde{Y}_{\rho, \mu}^{v}\{g(z) ; x\}\right]=\frac{x^{-\delta}}{\rho} \int_{0}^{\infty} H_{2,4}^{2,2} \\
& (\times)\left[\begin{array}{c}
(0,1),(-\kappa+\delta, 1) \\
z x \mid(0,1),\left(\frac{v}{\rho}, \frac{\mu}{\rho}\right),(\delta, 1),(-\gamma-\kappa, 1)
\end{array}\right] g(z) d z
\end{aligned}
$$

and

$$
\begin{aligned}
& I_{0, x}^{\gamma, \delta, \kappa}\left[\tilde{Y}_{\rho, \mu}^{v}\{g(z) ; x\}\right]=\frac{x^{-\delta}}{\rho} \int_{0}^{\infty} H_{3,3}^{1,3} \\
& (\times)\left[\begin{array}{c}
(0,1),(-\kappa+\delta, 1),\left(1-\frac{v}{\rho}, \frac{\mu}{\rho}\right) \\
z x \mid(0,1),(\delta, 1),(-\gamma-\kappa, 1)
\end{array}\right] g(z) d z
\end{aligned}
$$


To prove (4.9) we express the Saigo integral operator of the $\tilde{Y}$ transform with the help of (4.1), then we write transform by means of (1.1). Next, we interchange the order of $x$ integral with contour integral. We obtain the following form say $(\Delta)$

$$
\begin{aligned}
& \Delta=\frac{1}{\rho} \frac{1}{2 \pi i} \int_{L} \Gamma\left(\frac{v}{\rho}+\frac{\mu}{\rho} \zeta\right) \Gamma(-\zeta) \\
& (\times)\left\{\int_{0}^{\infty} z^{\zeta} g(z)\left[I_{+}^{\gamma, \delta, \kappa}\left(x^{\zeta}\right)\right] d z d \zeta\right\}
\end{aligned}
$$

Next, we solve inner $x$ integral using result (4.4) and reinterpreting the result in terms of $H$-function after some simplification, we get the desired result.

The proof of (4.10) can be obtained by proceeding on similar lines given to those given above using (4.8).

Theorem 4.2. If $D_{+}^{\gamma, \delta, \kappa}[g(z) ; x]$ and $D_{+}^{\gamma, \delta, \kappa}\left[\tilde{Y}_{\rho, \mu}^{v}\{g(z) ; x\}\right]$ exist, $g(z) \in L_{v, r}(0, \infty), s, v \in \mathbb{C}, \rho \in R, \rho \neq 0$ be such that $\operatorname{Re}(s)>$ $0, \operatorname{Re}(v+\mu s)>0$ and the condition of existence of $\tilde{Y}_{v}^{\rho, \mu}\{g(z) ; x\}$ defined by (1.1) are satisfied then

$$
\begin{aligned}
& D_{+}^{\gamma, \delta, \kappa}\left[\tilde{Y}_{\rho, \mu}^{v}\{g(z) ; x\}\right]=\frac{x^{\delta}}{\rho} \int_{0}^{\infty} H_{3,3}^{1,1} \\
& (\times)\left[\begin{array}{c}
\left(1-\frac{v}{\rho}, \frac{\mu}{\rho}\right),(0,1),(\kappa-\delta) \\
z x \mid(0,1),(\gamma+\kappa, 1),(-\delta, 1)
\end{array}\right] g(z) d z .
\end{aligned}
$$

Theorem 4.3. If $D_{-}^{\gamma, \delta, \kappa}[f(z) ; x]$ and $D_{-}^{\gamma, \delta, \kappa}\left[\tilde{Y}_{\rho, \mu}^{v}\{f(z) ; x\}\right]$ exist, $\operatorname{Re}(\gamma)>0$ and the condition of existence of $\tilde{Y}_{v}^{\rho, \mu}\{f(z) ; x\}$ defined by (1.1) are satisfied then

$$
\begin{aligned}
& D_{-}^{\gamma, \delta, \kappa}\left[\tilde{Y}_{\rho, \mu}^{v}\{f(z) ; x\}\right]=\frac{x^{\delta}}{\rho} \int_{0}^{\infty} H_{2,3}^{3,1} \\
& (\times)\left[\begin{array}{c}
\left(1-\frac{v}{\rho}, \frac{\mu}{\rho}\right),(\kappa-\delta, 1) \\
z x \mid(0,1),(-\delta, 1),(\gamma+\kappa, 1),(1, \mu)
\end{array}\right] f(z) d z .
\end{aligned}
$$

To prove (4.13) we write the Saigo differential operator [[5],p. 258, Eq.(7.12.45)] of the transform, then we write $\tilde{Y}$ transform by means of (1.1) \& (1.2).

Next, we interchange the order of $x$ integral with contour integral. We obtain the following form say $(\Delta)$

$$
\begin{aligned}
& \Delta=\frac{1}{\rho} \frac{1}{2 \pi i} \int_{L} \Gamma\left(\frac{v}{\rho}+\frac{\mu}{\rho} \zeta\right) \Gamma(-\zeta) \\
& (\times)\left\{\int_{0}^{\infty} z^{\zeta} g(z)\left[D_{+}^{\gamma, \delta, \kappa}\left(x^{\zeta}\right)\right] d z d \zeta\right\} .
\end{aligned}
$$

Next, we solve inner $x$ integral using result (4.7) and reinterpreting the result in terms of $H$-function after some simplification, we get the desired result.

The proof of (4.14) can be obtained by proceeding on similar lines given to those above using (4.8).

\section{$H$-function}

The $H$-function occurring in this paper will be defined and represented in the following manner [12].

$$
\begin{aligned}
& H_{p, q}^{m, n}\left[\begin{array}{c}
z \mid\left(a_{1}, \alpha_{1}\right), \ldots,\left(a_{n}, \alpha_{p}\right) \\
\left(b_{1}, \beta_{1}\right) \ldots\left(b_{q}, \beta_{q}\right)
\end{array}\right] \\
& =\frac{1}{2 \pi i} \int_{L} \frac{\prod_{j=1}^{m} \Gamma\left(b_{j}-\beta_{j} \zeta\right) \prod_{j=1}^{n} \Gamma\left(1-a_{j}+\alpha_{j} \zeta\right)}{\prod_{j=m+1}^{q} \Gamma\left(1-b_{j}+\beta_{j} \zeta\right) \prod_{j=n+1}^{p} \Gamma\left(a_{j}-\alpha_{j} \zeta\right)} z^{\zeta} d \zeta,
\end{aligned}
$$

where for the nature of contour $L$ and the other details of $H$-function defined by (4.15) we refer to the work cited above. The general class polynomials

The general class of polynomials, introduced by Srivastava [11] is given by

$$
S_{V}^{U}[x]=\sum_{R=0}^{[V / U]}(-V)_{U R} A_{V, R} \frac{x^{R}}{R !}, \quad V=0,1,2, \ldots
$$

where $U$ is an arbitrary positive integers, the coefficients $A_{V, R}$ are arbitrary constants, real or complex. On suitably specializing the coefficients $A_{V, R}$, yields a number of known polynomials as its special cases. These include among others the Cesaro polynomial, Konhauser polynomial, Brafman polynomial, Bedient polynomial, Bateman polynomial and several others.

\section{Conclusion}

In this paper, we have presented a pathway pathway integral transform associated with generalized Kratzel functions. The obtained result provided generalised forms of the known results earlier proved by Kratzel [9].

\section{References}

[1] A. Erdelyi, W. Magnus, F. Oberthettinger and F.G. Tricomi, Higher Transcendental Functions, Vol I, McGrawHill Book Company, New York, Toronto, London 1953.

[2] A.A. Kilbas and N. Sebastian, Generalised fractional differentiation of Bessel function of the first kind, Mathematica Balkanica, 22(2008), 323-346.

[3] A.A. Kilbas, and D. Kumar, On generalized Kratzel function, Integral Transforms and Special Functions, 20(2009), 835-846.

[4] A.A Kilbas, H.M. Srivastava and J.J. Trujillo, Theory and Applications of Fractional Differential Equations, Elsevier, Amsterdam, 2006.

[5] A.A. Kilbas, L. Rodriguez and J.J. Trujillo, Asymptotic representations for hypergeometric-Bessel type function and fractional integrals, J. Comput. Appl. Math., 149(2002), 469-487.

[6] A.A. Kilbas, R.K. Saxena and J.J. Trujillo, Kratzel function as a function of hypergeometric type, Frac. Calc. Appl. Anal., 9(2)(2006), 109-131.

[7] B Bonilla, A. A. Kilbas, M. Rivero, J. Rodriguez- L. Germa and J.J. Trujillo, Modified Bessel type function and solution of differential and integral equations, Indian J. Pure Appl. Math., 31(1)(2000), 93-109. 
[8] D. Kumar, $P$-transform, Integral Transform and Special Functions, 22(8)(2011), 603-616.

[9] E. Kratzel, Integral transformations of Bessel type, Proceeding of International Conference on Generalized Functions and Operational Calculus, Varna, 1975, 148155.

[10] H.J. Glaeske, A.A. Kilbas and M. Saigo, A modified Bessel-type integral transform and its compositions with fractional calculus operators on spaces $F P, \mu$ and $F P, \mu_{1}$, J. of Computational and Applied Mathematics, 118(2000), 151-168.

[11] H.M. Srivastava, A contour integral involving Fox's $H$ function, Indian J. Math., 14(1972), 1-6.

${ }^{[12]}$ H.M. Srivastava, K.C. Gupta and S.P. Goyal, The Hfunctions of One and Two Variables with Applications, South Asian Publishers, New Delhi and Madras, 1982.

[13] J.D.E. Konhauser, Biorthogonal polynomials suggested by the Lagurre polynomials, Pacific J. Math., 21(1967), 303-314.

[14] K.S. Miller and B Ross, An Introduction to the Fractional Calculus and Fractional Differential Equations, John Wiley and Sons Inc, 1993.

[15] M. Saigo, A remark on integral operators involving the Gauss hypergeometric functions, Math. Rep. Kyushu Univ., 11(1978), 135-143.

[16] N.S. Dernek, A.T. Dernek, and O.S. Yurekli, A Generalization of the Kratzel Function and Its Applications, New York, 2017.

[17] R Jain and P Kumawat, A Study of generalized pathway Transform, 2017,77-88.

[18] R.K.Saxena, On thermo nuclear reaction rate integrals through model, Rajasthan Ganita Parishad, (2012), 27 42.

[19] S.G. Samko, A.A. Kilbas and O.I. Marichev, Fractional Integrals and Derivatives: Theory and Applications, Gordon and Breach, New York, 1993.

[20] A.A. Inayat-Hussain, New properties of hypergeometric series derivable from Feynman integrals: II. A generalization of the $H$-function, J. Phys. A: Math. Gen., 20(1987), 4119-4128.

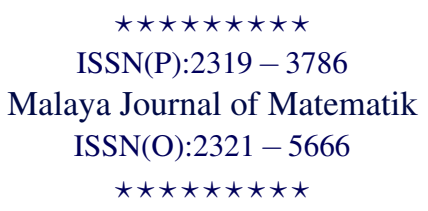

